UAB-FT-378

November, 1995

hep-ph/9511297

\title{
HIGGS TRIPLET EFFECTS IN PURELY LEPTONIC PROCESSES
}

\author{
J.A. Coarasa, A. Méndez and J. Solà \\ Grup de Física Teòrica and IFAE \\ Universitat Autònoma de Barcelona \\ 08193 Bellaterra (Barcelona), Catalonia, Spain
}

\begin{abstract}
We consider the effect of complex Higgs triplets on purely leptonic processes and survey the experimental constraints on the mass and couplings of their single and double charge members. Present day experiments tolerate values of the Yukawa couplings of these scalars at the level of the standard electroweak gauge couplings. We show that the proposed measurement of the ratio $R_{L C D}=\sigma\left(\nu_{\mu} e\right) /\left[\sigma\left(\bar{\nu}_{\mu} e\right)+\sigma\left(\nu_{e} e\right)\right]$ would allow to explore a large region of the parameter space inaccessible to the usual ratio $R=$ $\sigma\left(\nu_{\mu} e\right) / \sigma\left(\bar{\nu}_{\mu} e\right)$.
\end{abstract}


Although the Standard Model (SM) of the strong and electroweak interactions seems to agree very well with the experimental data, the Higgs sector still remains practically unexplored and one can therefore consider different extensions. Here we will focus our attention on Higgs triplets with non zero hypercharge. They are a common feature in models where the left-handed neutrinos acquire a Majorana mass through the Higgs mechanism. This is the case, for instance, of the classical version of the Left-Right Symmetric extension of the SM [1].

Higgs triplets under the standard $S U(2)_{L}$ gauge group have some very specific properties. Their vacuum expectation values have to be small in order not to spoil the agreement between the theoretical and the experimental values of the electroweak $\rho$ parameter [2]. Moreover, since they have two units of weak hypercharge, apart from the electrically neutral scalar $\left(h^{0}\right)$ there are also singly charged $\left(h^{+}\right)$and doubly charged $\left(h^{++}\right)$ones. This has an important phenomenological consequence, namely, that they can not couple to quarks and therefore their dominant effects are to be observed only in purely leptonic processes. Moreover, these effects are not necessarily small because the Higgs triplets do not participate in the generation of the lepton Dirac masses and therefore their Yukawa couplings can be large.

Another feature displayed by scalar triplets (and, more generally, by any unmixed higher dimension scalar multiplet) is that they exhibit equal masssquared spacing ordered by the scalar field electric charges [3, 4, This implies that, in general, $m_{h^{++}}>m_{h^{+}}$.

Several experimental constraints on the couplings and masses of the $h^{++}$ have been considered in the literature [4, 5, 6, 7]. They include the constraints arising from the value of the anomalous magnetic moment of the electron and the muon, the angular distribution in the Bhabha scattering and the experimental limits on muonium-antimuonium transitions and non standard muon decays. The effects of the singly charged member of the triplet, $h^{+}$, have not been considered so extensively. The existence of such field would affect the purely leptonic neutrino processes and precise measurements in this sector, like those of the CHARM II collaboration [8], impose additional constraints. In this respect, there has been a proposal by the Large Cerenkov Detector (LCD) Project collaboration [9] of an experiment at LAMPF that 
would allow the measurement of the ratio

$$
R_{L C D} \equiv \frac{\sigma\left(\nu_{\mu} e\right)}{\sigma\left(\bar{\nu}_{\mu} e\right)+\sigma\left(\nu_{e} e\right)},
$$

with a precision of $2 \%$ (corresponding to a $0.9 \%$ precision in the measurement of $\left.\sin ^{2} \theta_{W}\right)$. Such high precision is due to the particular setup of this experiment which will be almost free of systematic uncertainties [9], making it competitive with the standard CHARM II ratio $R=\sigma\left(\nu_{\mu} e\right) / \sigma\left(\bar{\nu}_{\mu} e\right)$ from which the value of $\sin ^{2} \theta_{W}$ is obtained with an accuracy at the level of $3.6 \%$. Therefore, the ratio $R_{L C D}$ could be a suitable tool to probe physics beyond the SM. This is the case, for instance, of supersymmetric effects [10]. The existence of $h^{ \pm}$would also affect this ratio and more stringent bounds on its couplings and mass might be imposed from the measured value.

In this paper we shall determine the region in the parameter space where the effects of the $h^{ \pm}$bosons could be observed in the measurement of $R_{L C D}$. This requires first an updating of the experimental constraints on the $h^{ \pm}$mass and Yukawa couplings. At the end we shall comment on the implications for the $h^{ \pm \pm}$mass and couplings.

The term of the lagrangian describing the interaction of the triplet with the leptons can be written as

$$
\mathcal{L}=i \sum_{i, j=e, \mu, \tau} g_{i j}\left(\Psi_{i L}^{T} \mathcal{C} \tau_{2} \Delta \Psi_{j_{L}}\right)+\text { h.c. }
$$

where $\mathcal{C}$ is the charge conjugation matrix, $\Psi_{i L}$ are the standard lepton doublets and $\Delta$ is the scalar triplet written in the usual matrix form,

$$
\Delta=\left(\begin{array}{cc}
h^{+} / \sqrt{2} & h^{++} \\
h^{0} & -h^{+} / \sqrt{2}
\end{array}\right) .
$$

The interactions described by the lagrangian of eq. (2) do not conserve the lepton family numbers in general. They do conserve, however, the total lepton number, $L$, if the value $L=-2$ is assigned to the scalar triplet $\Delta$.

We assume the coupling constants $g_{i j}$ to be real. The relevant Feynman rules are shown in fig. 1 in terms of $h_{i j} \equiv\left(g_{i j}+g_{j i}\right) / 2$. (Notice that, by definition, $h_{i j}=h_{j i}$.)

Defining $\delta_{i j} \equiv h_{i j} / m_{h^{+}}$, we list now the constraints on $\delta_{i j}$ arising from different experimental measurements. 


\section{a. Restrictions from the decay $\mu \rightarrow e \gamma$}

Parametrizing the $\mu e \gamma$ vertex in the usual way,

$$
\Lambda^{\lambda}+\Lambda_{A}^{\lambda} \gamma_{5}
$$

with

$$
\begin{aligned}
& \Lambda^{\lambda}=F_{1} \gamma^{\lambda}+\frac{F_{2}}{m_{\mu}+m_{e}} i \sigma^{\lambda \nu} q_{\nu}+\frac{F_{3}}{m_{\mu}+m_{e}} q^{\lambda}, \\
& \Lambda_{A}^{\lambda}=G_{1} \gamma^{\lambda}+\frac{G_{2}}{m_{\mu}+m_{e}} i \sigma^{\lambda \nu} q_{\nu}+\frac{G_{3}}{m_{\mu}+m_{e}} q^{\lambda},
\end{aligned}
$$

the decay width, $\Gamma(\mu \rightarrow e \gamma)$, in the limit $m_{e}<<m_{\mu}$ can be written as

$$
\Gamma(\mu \rightarrow e \gamma)=\frac{\alpha m_{\mu}}{2}\left(\left|F_{2}\right|^{2}+\left|G_{2}\right|^{2}\right)
$$

and the corresponding branching ratio is

$$
B(\mu \rightarrow e \gamma)=\frac{96 \pi^{2} \alpha}{G_{F}^{2} m_{\mu}^{4}}\left(\left|F_{2}\right|^{2}+\left|G_{2}\right|^{2}\right)
$$

The $h^{-}$contribution to $F_{2}, G_{2}$ arising from the diagram of fig. 2.a is

$$
F_{2}=G_{2}=\frac{m_{\mu}^{2}}{192 \pi^{2}}\left[\delta_{e \mu}\left(\delta_{e e}+\delta_{\mu \mu}\right)+\delta_{e \tau} \delta_{\mu \tau}\right]
$$

From the experimental bound [11]

$$
B(\mu \rightarrow e \gamma)<4.9 \times 10^{-11} \quad(90 \% \text { C.L. }),
$$

using eqs.(3) and (4) we obtain

$$
\left|\delta_{e \mu}\left(\delta_{e e}+\delta_{\mu \mu}\right)+\delta_{e \tau} \delta_{\mu \tau}\right|<4.2 \times 10^{-8} \mathrm{GeV}^{-2} \quad \text { (90\% C.L.) } .
$$

b. Restrictions from the decay $\mu \rightarrow e \nu_{e} \bar{\nu}_{\mu}$ and neutrino oscillation experiments

The existence of $h^{-}$would allow the non standard decay $\mu \rightarrow e \nu_{e} \bar{\nu}_{\mu}$ through the diagram of fig. 2.b. The corresponding width is

$$
\Gamma\left(\mu \rightarrow e \nu_{e} \bar{\nu}_{\mu}\right)=\frac{m_{\mu}^{5}}{1536 \pi^{3}} \delta_{e e^{2}}^{2} \delta_{\mu \mu}^{2}
$$


From the experimental limit 11

$$
\frac{\Gamma\left(\mu \rightarrow e \nu_{e} \bar{\nu}_{\mu}\right)}{\Gamma\left(\mu \rightarrow e \bar{\nu}_{e} \nu_{\mu}\right)}<0.012 \quad(90 \% \text { C.L. })
$$

the following bound can be obtained

$$
\left|\delta_{e e} \delta_{\mu \mu}\right|<3.6 \times 10^{-6} \mathrm{GeV}^{-2} \quad \text { (90\% C.L.) } .
$$

(If we further assume $e-\mu$ universality then we would have $\left|\delta_{e e}\right|=\left|\delta_{\mu \mu}\right|<$ $1.9 \times 10^{-3}$.)

Recently, it has been pointed out in ref. [12] that the results of the KARMEN experiment [13] on neutrino oscillations lead to the bound

$$
\left(\frac{G_{N}}{G_{F}}\right)^{2}<3.1 \times 10^{-3} G_{e V}^{-2} \quad(90 \% \text { C.L. }),
$$

where $G_{F}$ is the Fermi constant and $G_{N}$ is the coupling constant of an effective four fermion interaction of the form

$$
\mathcal{L}=G_{N}\left(\bar{\mu} \gamma_{\lambda} P_{L} e\right)\left(\bar{\nu}_{\mu} \gamma^{\lambda} P_{L} \nu_{e}\right)+\text { h.c. },
$$

with $P_{L}=\left(1-\gamma_{5}\right) / 2$. The amplitude of the diagram of fig. 2.b can be written this form after a suitable Fierz reordering with $G_{N} \equiv \delta_{e e} \delta_{\mu \mu}$. Then, from eq. (5) we can obtain the more restrictive bound

$$
\left|\delta_{e e} \delta_{\mu \mu}\right|<6.5 \times 10^{-7} \mathrm{GeV}^{-2} \quad \text { (90\% C.L.) } .
$$

(Again, assuming $e-\mu$ universality we would have $\left|\delta_{e e}\right|=\left|\delta_{\mu \mu}\right|<8.1 \times 10^{-4}$.)

\section{c. Restrictions from anomalous magnetic moments}

By explicit calculation of the diagrams of fig. 2.c we find the following contribution to the anomalous magnetic moment, $a_{i} \equiv(g-2) / 2$, of the charged lepton $i$,

$$
\Delta a_{i}=-\frac{m_{i}^{2}}{48 \pi} \sum_{j=e, \mu, \tau} \delta_{i j}^{2} .
$$

The theoretical values of $a_{e}$ and $a_{\mu}$ are 14

$$
\begin{aligned}
& a_{e}^{t h}=(1159652460 \pm 127 \pm 75) \times 10^{-12}, \\
& a_{\mu}^{t h}=(11659202 \pm 20) \times 10^{-10},
\end{aligned}
$$


and the corresponding experimental values are [11]

$$
\begin{aligned}
& a_{e}^{e x p}=(1159652193 \pm 10) \times 10^{-12}, \\
& a_{\mu}^{e x p}=(11659230 \pm 84) \times 10^{-10} .
\end{aligned}
$$

From these values we can obtain the $90 \%$ C.L. intervals for any non standard contribution to $a_{i}$ 円,

$$
\begin{gathered}
-5.1 \times 10^{-10}<\Delta a_{e}<-0.2 \times 10^{-10}, \\
-1.1 \times 10^{-8}<\Delta a_{\mu}<1.7 \times 10^{-8} .
\end{gathered}
$$

Since the contribution given by eq. (7) is negative, the following bounds apply,

$$
\left|\Delta a_{e}\right|<5.1 \times 10^{-10}, \quad\left|\Delta a_{\mu}\right|<1.1 \times 10^{-8} .
$$

Then, the following restrictions are obtained,

$$
\begin{aligned}
& \sum_{j=e, \mu, \tau} \delta_{e j}{ }^{2}<0.29 \mathrm{GeV}^{-2} \quad \text { (90\% C.L.) } \\
& \sum_{j=e, \mu, \tau} \delta_{\mu j}^{2}<1.5 \times 10^{-4} \mathrm{GeV}^{-2} \quad \text { (90\% C.L.) },
\end{aligned}
$$

from which we can infer the following bounds,

$$
\begin{aligned}
\left|\delta_{e e}\right|,\left|\delta_{e \tau}\right| & <0.54 \mathrm{GeV}^{-1}
\end{aligned}
$$

\section{d. Restrictions from the value of $\sin ^{2} \theta_{W}$ measured in purely leptonic neutrino interactions}

Using the ratio $R=\sigma\left(\nu_{\mu} e\right) / \sigma\left(\bar{\nu}_{\mu} e\right)$, the CHARM II Collaboration [8] has been able to obtain a precise value of $\sin ^{2} \theta_{W}$ from purely leptonic $\nu_{\mu} e$ and $\bar{\nu}_{\mu}$ e collisions,

$$
x_{W} \equiv \sin ^{2} \theta_{W}=0.2324 \pm 0.0083 .
$$

The SM tree level amplitude for the $\nu_{\mu} e$ scattering is given by

$$
\mathcal{M}=i \frac{G_{F}}{\sqrt{2}} \bar{e} \gamma_{\lambda}\left[P_{L}-2 x_{W}\right] e \times \bar{\nu} \gamma^{\lambda}\left(1-\gamma_{5}\right) \nu
$$

\footnotetext{
${ }^{1}$ They are given by $a^{e x p}-a^{t h} \pm 1.645\left(\sigma_{e x p}^{2}+\sigma_{t h}^{2}\right)^{1 / 2}$.
} 
A similar expression with straightforward modifications affecting only the last factor of the r.h.s. of eq. (11) can be written for the $\bar{\nu}_{\mu} e$ amplitude.

The two $h^{-}$exchanging diagrams of fig. 2.d add new contributions to the $\nu_{\mu} e$ and $\bar{\nu}_{\mu} e$ amplitudes respectively. With a suitable Fierz transformation one can readily see that the net effect of these $h^{-}$contributions consists in replacing the term inside the square brackets of eq. (11) by

$$
\begin{aligned}
P_{L}-2 x_{W} & \rightarrow\left(1+\frac{\delta_{e \mu}{ }^{2}}{\sqrt{2} G_{F}}\right) P_{L}-2 x_{W} \\
& \simeq\left(1+\frac{\delta_{e \mu}{ }^{2}}{\sqrt{2} G_{F}}\right)\left[P_{L}-2 x_{W}\left(1-\frac{\delta_{e \mu}{ }^{2}}{\sqrt{2} G_{F}}\right)\right] .
\end{aligned}
$$

This replacement induces an apparent shift of $x_{W}$ and the $\rho$ parameter given by

$$
\Delta x_{W} \simeq-\frac{x_{W} \delta_{e \mu}{ }^{2}}{\sqrt{2} G_{F}}, \quad \Delta \rho=\frac{\delta_{e \mu}{ }^{2}}{\sqrt{2} G_{F}} .
$$

The agreement between the results of the CHARM II Collaboration with those obtained from semi-leptonic $\nu$ interactions (where $h^{ \pm}$effects would be absent) suggests that $\delta_{e \mu}{ }^{2} / \sqrt{2} G_{F}$ is small (of the order of a few percent at most) and thus justifies the use of the approximate form given by the last expression of eq. (12).

The value given by eq. (10) coincides with the theoretical prediction, $x_{W}^{t h}=0.2324 \pm 0.0012$ (see, e.g., ref. [15]). The $90 \%$ C.L. interval for $\Delta x_{W}$ is therefore

$$
\left|\Delta x_{W}\right|<0.0138
$$

from which we obtain

$$
\left|\delta_{e \mu}\right|<9.9 \times 10^{-4} \mathrm{GeV}^{-1} \quad(90 \% \text { C.L. }) .
$$

A similar analysis can be carried out in the case of $\nu_{e} e$ collisions. The experimental study has been done at LAMPF [16] and the obtained experimental value for $x_{W}$ is

$$
x_{W}=0.249 \pm 0.063 .
$$

In this case the SM tree level amplitude can be conveniently written as

$$
\mathcal{M}=i \frac{G_{F}}{\sqrt{2}} \bar{e} \gamma_{\lambda}\left[-2 P_{L}+\left(P_{L}-2 x_{W}\right)\right] e \times \bar{\nu} \gamma^{\lambda}\left(1-\gamma_{5}\right) \nu
$$


The effect of the $h^{-}$contribution is again the substitution (12) with $\delta_{e \mu}$ replaced by $\delta_{e e}$. The extra term, $-2 P_{L}$, (compared to the $\nu_{\mu} e$ case) is due to the $W$ exchange contribution and is unaffected by the $h^{-}$exchange. The apparent shift on $x_{W}$ is now

$$
\Delta x_{W} \simeq-\frac{x_{W} \delta_{e e}^{2}}{\sqrt{2} G_{F}},
$$

and the $90 \%$ C.L. interval for $\Delta x_{W}$ is

$$
-0.09<\Delta x_{W}<0.12,
$$

which leads to the following bound

$$
\left|\delta_{e e}\right|<2.5 \times 10^{-3} \mathrm{GeV}^{-1} \quad \text { (90\% C.L.) } .
$$

An alternative approach in order to obtain bounds on non SM physics from the measured values of the $\sigma\left(\nu_{e} e\right)$ is described in ref. [16]. If we write

$$
\frac{d \sigma\left(\nu_{e} e\right)}{d y}=\sigma_{0}\left[A+B(1-y)+C(1-y)^{2}\right],
$$

where $\sigma_{0}=G_{F}^{2} s / 4 \pi$, the $h^{-}$exchange diagram adds the following contribution to the quantity $A$,

$$
\Delta A=-\left(2+4 x_{W}\right) \frac{\delta_{e e}^{2}}{\sqrt{2} G_{F}},
$$

and from the experimental value of $\sigma\left(\nu_{e} e\right)$ the following bound on $\delta_{e e}$ can be derived (see ref. [16] for details?2),

$$
\left|\delta_{e e}\right|<7.2 \times 10^{-3} \mathrm{GeV}^{-1} \quad(90 \% \text { C.L. }) \text {, }
$$

which is less restrictive than the bound of eq. (14).

The restrictions imposed by the above observables on $\delta_{e e}, \delta_{\mu \mu}$ and $\delta_{e \mu}$ are summarized in table 1 where only the most restrictive ones, given by eqs. (6),

\footnotetext{
${ }^{2}$ The fact that the $h^{-}$exchange diagram modifies the quantity $A$ is due to the helicity structure in the lagrangian of eq. (2). A charged Higgs belonging to a doublet would, instead, contribute to $C$ (see ref. [16]).
} 
(9), (13) and (14), are listed. The combined bounds on $\delta_{i j}$ are also shown in fig. 3 .

We now turn to the ratio $R_{L C D}$ defined in eq. (11). Introducing the quantities

$$
\begin{aligned}
& \epsilon_{-}=\frac{1}{2}\left(1-2 x_{W}\right), \\
& \epsilon_{-}^{\prime}=\frac{1}{2}\left(-1-2 x_{W}\right), \\
& \epsilon_{+}=\epsilon_{+}^{\prime}=-x_{W},
\end{aligned}
$$

the tree level SM value can be written as

$$
R_{L C D}^{0}=\frac{\epsilon_{-}^{2}+\frac{1}{3} \epsilon_{+}^{2}}{\epsilon_{+}^{2}+\frac{1}{3} \epsilon_{-}^{2}+\epsilon_{-}^{\prime 2}+\frac{1}{3} \epsilon_{+}^{\prime 2}}=0.142 .
$$

This value is modified by the presence of non SM physics (in our case, the $h^{-}$interactions) as well as by SM radiative corrections. The dominant $h^{-}$ contributions to the cross sections appearing in eq. (11) are given by the two diagrams of fig. 2.d and the first one involving the electron neutrino. The effect of these $h^{-}$exchange contributions is taken into account by simply replacing in eq. (15) the quantities $\epsilon_{ \pm}$and $\epsilon_{ \pm}^{\prime}$ by $\tilde{\epsilon}_{ \pm}$and $\tilde{\epsilon}_{ \pm}^{\prime}$ respectively, where

$$
\begin{aligned}
& \tilde{\epsilon}_{+}=\epsilon_{+}, \quad \tilde{\epsilon}_{-}=\epsilon_{-}+\frac{1}{2} \frac{\delta_{e \mu}{ }^{2}}{\sqrt{2} G_{F}}, \\
& \tilde{\epsilon}_{+}^{\prime}=\epsilon_{+}^{\prime}, \quad \tilde{\epsilon}_{-}^{\prime}=\epsilon_{-}^{\prime}+\frac{1}{2} \frac{\delta_{e e}{ }^{2}}{\sqrt{2} G_{F}} .
\end{aligned}
$$

Thus, we have

$$
R_{L C D}=\frac{\tilde{\epsilon}_{-}^{2}+\frac{1}{3} \tilde{\epsilon}_{+}^{2}}{\tilde{\epsilon}_{+}^{2}+\frac{1}{3} \tilde{\epsilon}_{-}^{2}+\tilde{\epsilon}_{-}^{\prime 2}+\frac{1}{3} \tilde{\epsilon}_{+}^{2}}+\Delta R_{L C D}^{R . C .},
$$

where $\Delta R_{L C D}^{R . C .}$ is the effect of the SM radiative corrections which have been computed in ref. [17]. The result is

$$
\Delta R_{L C D}^{R . C .}=0.036 \times R_{L C D}^{0}=5.1 \times 10^{-3} .
$$


In fig. 3.b we plot the contour lines of $R_{L C D} / R_{L C D}^{S M}$, where

$$
R_{L C D}^{S M} \equiv R_{L C D}^{0}+\Delta R_{L C D}^{R . C .}=0.147,
$$

is the SM prediction (including radiative corrections).

We observe that in a large part of the allowed region in the $\left|\delta_{e e}\right|-\left|\delta_{e \mu}\right|$ plane the deviations of the ratio $R_{L C D}$ from the SM prediction are larger than $2 \%$ and therefore detectable. For some (allowed) values of $\delta_{e e}$ and $\delta_{e \mu}$ these deviations can be even larger than $50 \%$ (in the top right corner of the allowed region of fig. 3.b the value of $R_{L C D}$ would double the SM one). On the other hand, if no deviations from the SM were observed the region above the 1.02 curve would be ruled out.

If the inequality $m_{h^{+}}<m_{h^{+}+}$holds, as it happens in practically all Higgs triplet models, both the measurement of $R_{L C D}$ and the bounds on $\delta_{i j}$ described above would also affect the restrictions on the mass and couplings of the doubly charged scalar, $h^{++}$. Indeed, defining $\delta_{i j}^{\prime} \equiv h_{i j} / m_{h^{++}}$, if the singly charged scalars are lighter than the doubly charged ones then $\delta_{i j}^{\prime}<\delta_{i j}$ and therefore any upper bound of $\delta_{i j}$ would be also an upper bound of $\delta_{i j}^{\prime}$.

The direct bounds on $\delta_{i j}^{\prime}$ (without assuming the inequality $m_{h^{+}}<m_{h^{++}}$) are obtained from the experimental results on $(g-2)$, Bhabha scattering, muonium-antimuonium $(M \bar{M})$ transitions and the decay $\mu^{-} \rightarrow e^{-} e^{+} e^{-}$. They are summarized in the left half of table 2 .

The $(g-2)$ bound is obtained by applying eq. (8) to the $h^{++}$contribution to $a_{i}$ given by [4],

$$
\Delta a_{i}=-\frac{m_{i}^{2}}{6 \pi} \sum_{j=e, \mu, \tau} \delta_{i j}^{\prime 2} .
$$

The resulting inequalities are

$$
\begin{aligned}
\left|\delta_{e e}^{\prime}\right|,\left|\delta_{e \tau}^{\prime}\right| & <0.19 \mathrm{GeV}^{-1}
\end{aligned}
$$

The remaining bounds are discussed in ref. [6][ ]

If $m_{h^{+}}<m_{h^{++}}$, the bounds of table 1 became also bounds on $\delta_{i j}^{\prime}$. We show them in the right half of table 2 . In the case of $\delta_{\mu \mu}^{\prime}$, the slight improvement of the bound is obtained by adding the $h^{+}$and $h^{++}$contributions to

\footnotetext{
${ }^{3}$ The $M \bar{M}$ bound has been updated according to the new experimental limit given in ref. [11]. The Bhabha scattering and $\mu \rightarrow 3 e$ bounds were also discussed in ref. [4]. Notice that our $h_{e e}, h_{\mu \mu}$ and $h_{e \mu}$ are respectively the $g_{e e}, g_{\mu \mu}$ and $g_{e \mu} / 2$ of ref. [6].
} 
$a_{i}$, given by eqs. (7) and (16) respectively, and using the inequality $\delta_{i j}^{\prime}<\delta_{i j}$. We have then

$$
\frac{3 m_{i}^{2}}{16 \pi} \sum_{j=e, \mu, \tau} \delta_{i j}^{\prime 2}<\left|\Delta a_{i}^{\left(h^{+}\right)}+\Delta a_{i}^{\left(h^{++}\right)}\right|,
$$

with the r.h.s. satisfying the bounds of eq. (8).

The $\mu \rightarrow 3 e$ bound on $\left|\delta_{e e}^{\prime} \delta_{e \mu}^{\prime}\right|$ appearing in the last line of table 2 is, by far, the most restrictive one and implies that either $m_{h^{++}}$is very large or $h_{e e} h_{e \mu}$ is very small. If the couplings $h_{i j}$ are not small compared to, say, the $S U(2)_{L}$ gauge coupling $g$, then $h^{++}$must be very massive. In fact, if $h_{e e} h_{e \mu} \sim g^{2}$ the $h^{++}$mass would be extremely large $(\sim 100 \mathrm{TeV})$. The same applies to $m_{h^{+}}$and $m_{h^{0}}$, for if the three masses were very different there would be unacceptable contributions to the electroweak $\rho$ parameter [4]. As a result, the quantities $\delta_{i j}$ and $\delta_{i j}^{\prime}$ would be very small and Higgs triplet effects at the tree level would be hard to detect. Nevertheless, there could still be sizeable one-loop effects through (oblique) contributions to the $\rho$ parameter.

If, instead, $h_{e e} h_{e \mu}$ is very small, one can consider two situations. One possibility is that both couplings are very small as it happens with the Yukawa couplings of the SM Higgs to the first two fermion generations, which are several orders of magnitude smaller than the gauge couplings, a fact that is considered an unnatural feature of the SM. Another theoretically more appealing possibility is that $h_{e \mu}=0$ (i.e., that the Higgs triplet interactions are diagonal in lepton flavour) and $h_{e e} \sim h_{\mu \mu} \sim g$. Indeed, one can have this type of scenario compatible with all the bounds of tables 11 and 2 . For instance, if $m_{h^{+}} \lesssim m_{h^{++}} \sim 500 \mathrm{GeV}$, one could have $h_{e e} h_{\mu \mu} \sim g^{2} / 5$ while fulfilling the $\rho$ parameter constraint. Thus, extended Higgs sectors as the one considered here are specially attractive since they can be "natural", entailing a potentially rich phenomenology beyond the SM. 


\section{Acknowledgements}

Work supported in part by the CICYT research project AEN95-0882. J.S. is grateful to W.C. Louis and D.H. White for their hospitality at LAMPF and for useful discussions on the LCD project.

\section{References}

[1] J.C. Pati and A. Salam., Phys. Rev. D10 (1974) 275; R.N. Mohapatra and J.C. Pati, Phys. Rev. D11 (1975) 566, 2558; G. Senjanovic and R.N. Mohapatra, Phys. Rev. D12 (1975) 1502; R.N. Mohapatra and G. Senjanovic, Phys. Rev. Lett. 44 (1980) 912 and Phys. Rev. D23 (1981) 165.

[2] J.A. Grifols and A. Méndez, Phys. Rev. D22 (1980) 1725.

[3] A. Méndez and T.J. Weiler, Vanderbilt Univ. VAND-TH preprint, in preparation.

[4] J.F. Gunion, J. Grifols, A. Méndez, B. Kaiser and F. Olness, Phys. Rev. D40 (1989) 1546.

[5] J. Leveille, Nucl. Phys. B137 (1978) 63; V. Barger, H. Baer, W.Y. Keung and R.J.N. Phillips, Phys. Rev. D26 (1982) 218.

[6] M.L. Swartz, Phys. Rev. D40 (1989) 1521.

[7] P. Herczeg and R.N. Mohapatra, Phys. Rev. Lett. 69 (1992) 2475.

[8] P. Vilain et al., CHARM II Collab., Phys. Lett. B335 (1994) 246.

[9] A Proposal for a Precision Test of the Standard Model by NeutrinoElectron Scattering (Large Cerenkov Detector Project), Los Alamos report LA-11300-P (1988).

[10] J.A. Coarasa, A. Méndez and J. Solà, UAB-FT preprint, in preparation; for early studies on supersymmetric effects on leptonic processes see J.A. Grifols and J. Solà, Nucl. Phys. B253 (1985) 47; see also J. Solà, in Phenomenological Aspects of Supersymmetry, Lecture Notes in Physics 405, ed. by W. Hollik, R. Rückl and J. Wess (Springer-Verlag, 1992). 
[11] L. Montanet et al., Particle Data Group, Phys. Rev. D50 (1994) 1173.

[12] Y. Grossman, Weizmann Inst. preprint WIS-95/30/Jul-PH (1995).

[13] G. Drexlin et al., KARMEN Collaboration, Progr. Part. Nucl. Phys. 32 (1994) 375; Nucl Phys. (Proc. Suppl.) B38 (1995) 235.

[14] T. Kinoshita and W.B. Lindquist, Phys. Rev. Lett. 47 (1981) 1573; T. Kinoshita, B. Nizic and Y. Okamoto, Phys. Rev. Lett. 52 (1984) 717.

[15] W. Hollik, in Proc. of the Tennessee Int. Symp. on Radiative Corrections, Gatlinburg, TN; ed. by B.F.L. Ward, (World Scientific, 1995).

[16] R.C. Allen et al., Phys. Rev. D47 (1993) 11.

[17] S. Sarantakos, A. Sirlin and W.J. Marciano, Nucl. Phys. B217 (1983) 84.

\section{Figure Captions}

Fig. 1: Feynman rules corresponding to the interactions described by the lagrangian of eq. (2). Each arrow indicates one unit of total lepton number.

Fig. 2: Diagrams contributing to the different observables described in the text: a) $\mu^{-} \rightarrow e^{-} \gamma$; b) $\mu^{-} \rightarrow \bar{\nu}_{\mu} e^{-} \nu_{e}$; c) anomalous magnetic moment of the electron and muon; and d) $\nu_{\mu} e, \bar{\nu}_{\mu} e$ and $\nu_{e} e$ collisions.

Fig. 3: Allowed regions (white zones) in the $\left|\delta_{e e}\right|-\left|\delta_{\mu \mu}\right|$ plane (a) and $\left|\delta_{e e}\right|-\left|\delta_{e \mu}\right|$ plane (b). In the latter we also show the contour lines for the ratio $R_{L C D} / R_{L C D}^{S M}$ as predicted by the Higgs triplet model. 


\begin{tabular}{|c|c|}
\hline $90 \%$ C.L. bound & Process \\
\hline \hline$\left|\delta_{e e}\right|<2.5 \times 10^{-3} \mathrm{GeV}^{-1}$ & $\nu_{e} e$ \\
$\left|\delta_{e \mu}\right|<9.9 \times 10^{-4} \mathrm{GeV}^{-1}$ & $\nu_{\mu} e, \bar{\nu}_{\mu} e$ \\
$\left|\delta_{\mu \mu}\right|<1.2 \times 10^{-2} \mathrm{GeV}^{-1}$ & $(g-2)_{\mu}$ \\
$\left|\delta_{e e} \delta_{\mu \mu}\right|<6.5 \times 10^{-7} \mathrm{GeV}^{-2}$ & $\nu$ osc. \\
\hline
\end{tabular}

Table 1: Best bounds on $\delta_{i j}$.

\begin{tabular}{|c|c||c|c|}
\hline $\begin{array}{c}90 \% \text { C.L. bound } \\
\text { (general) }\end{array}$ & Process & $\begin{array}{c}90 \% \text { C.L. bound } \\
\text { (assuming } m_{h^{+}}<m_{h^{++}} \text {) }\end{array}$ & Process \\
\hline \hline$\left|\delta_{e e}^{\prime}\right|<2.8 \times 10^{-3} \mathrm{GeV}^{-1}$ & Bhabha & $\left|\delta_{e}^{\prime}\right|<2.5 \times 10^{-3} \mathrm{GeV}^{-1}$ & $\nu_{e} e$ \\
$\left|\delta_{e \mu}^{\prime}\right|<4.3 \times 10^{-3} \mathrm{GeV}^{-1}$ & $(g-2)_{\mu}$ & $\left|\delta_{e}^{\prime}\right|<9.9 \times 10^{-4} \mathrm{GeV}^{-1}$ & $\nu_{\mu} e, \bar{\nu}_{\mu} e$ \\
$\left|\delta_{\mu \mu}^{\prime}\right|<4.3 \times 10^{-3} \mathrm{GeV}^{-1}$ & $(g-2)_{\mu}$ & $\left|\delta_{\mu \mu}^{\prime}\right|<4.1 \times 10^{-3} \mathrm{GeV}^{-1}$ & $(g-2)_{\mu}$ \\
$\left|\delta_{e}^{\prime} \delta_{\mu \mu}^{\prime}\right|<8.6 \times 10^{-6} \mathrm{GeV}^{-2}$ & $M \bar{M}$ & $\left|\delta_{e e}^{\prime} \delta_{\mu \mu}^{\prime}\right|<6.5 \times 10^{-7} \mathrm{GeV}^{-2}$ & $\nu$ osc. \\
$\left|\delta_{e e}^{\prime} \delta_{e \mu}^{\prime}\right|<2.3 \times 10^{-11} \mathrm{GeV}^{-2}$ & $\mu \rightarrow 3 e$ & $\left|\delta_{e e}^{\prime} \delta_{e \mu}^{\prime}\right|<2.3 \times 10^{-11} \mathrm{GeV}^{-2}$ & $\mu \rightarrow 3 e$ \\
\hline
\end{tabular}

Table 2: Best bounds on $\delta_{i j}^{\prime}$. 



Fig. 1 


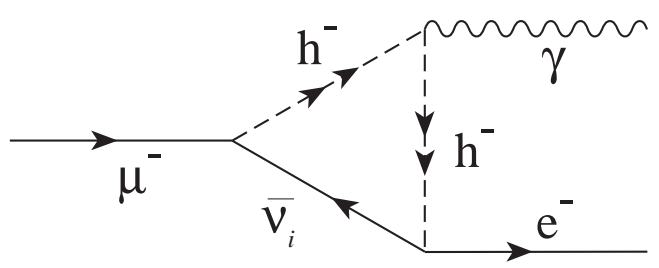

(a)

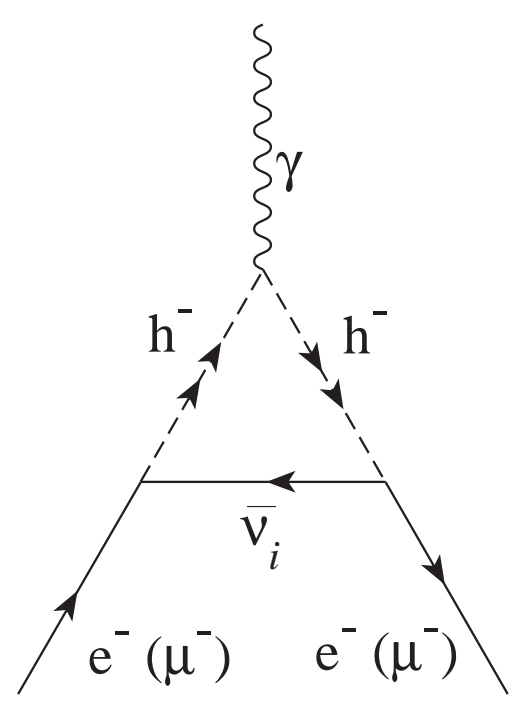

(c)



(b)


(d)

Fig. 2 

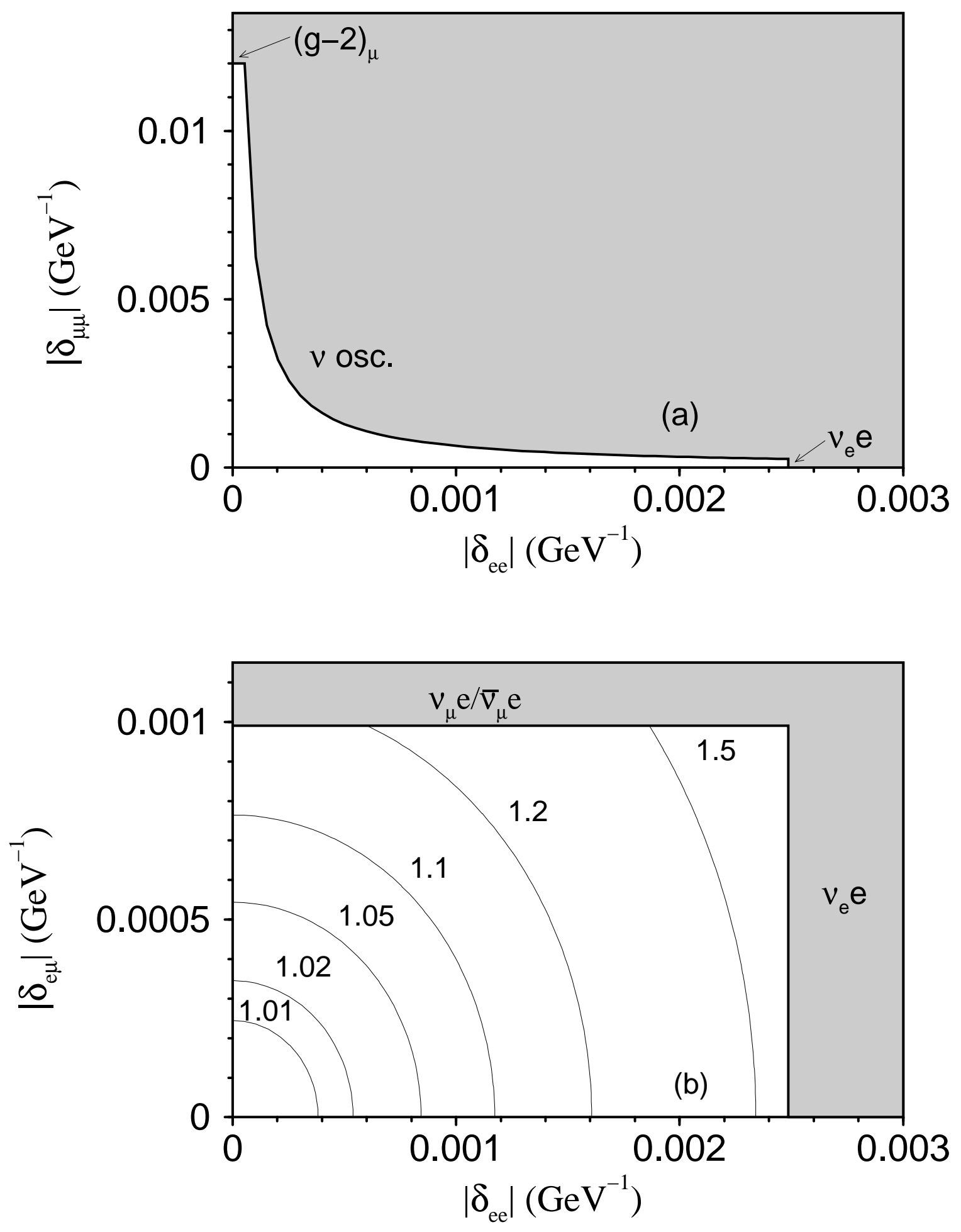

Fig. 3 